\title{
Improve the Effective Data Analysis Method of Enterprise E-Commerce Marketing
}

\author{
Jianhao He \\ Department of Business Administration, Ningbo Polytechnic, Ningbo City Zhejiang Province, 315800, China
}

\begin{abstract}
Under the background of Internet big data, data analysis is one of the important tools for enterprises to implement personalized e-commerce marketing and optimize their e-commerce service mode. It can not only improve the marketing ability of enterprises, but also innovate the e-commerce marketing mode. In terms of the construction of personalized and accurate e-commerce marketing service system, data analysis tools can be used to comprehensively improve the e-commerce marketing effect of enterprise products from three perspectives: product marketing, channel marketing and promotion marketing. In terms of the innovation of e-commerce service mode, an enterprise e-commerce information platform within the scope of supply chain network can be built, and the evaluation system and product traceability system of e-commerce service can be improved, so as to provide consumers with more convenient and comprehensive online services.
\end{abstract}

Key words: data analysis, enterprise, e-commerce, marketing

\section{Introduction}

Under the traditional economic model, marketing mainly relies on various print and TV media, which covers a narrow range of potential consumer groups, poor marketing effect and high advertising cost. Especially for enterprise products, the attention is even lower. The marketing activities based on the e-commerce model are mainly carried out through the network, in a variety of ways. More importantly, it can save a lot of advertising costs and reduce the burden of marketing costs of enterprises. Third, e-commerce marketing is more abundant in service mode, which can provide online consulting and video display of related products, and can also provide a service mode combining online consulting and offline experience to meet the experience needs of some consumers. With the increasing importance of e-commerce marketing mode, in order to further reduce marketing costs and improve marketing effects, enterprises need to accurately grasp consumers' consumption habits and preferences based on marketing data analysis, so as to provide consumers with more accurate and personalized services. By analyzing the role of big data in e-commerce marketing, this paper constructs a personalized marketing strategy system and innovative service mode based on data analysis[1].

\section{The function of data analysis in enterprise e-commerce marketing}

The product sales activities and sales performance of an enterprise are influenced by a variety of factors, including the external market environment, the stability of the supply chain, the overall scale of the livestock industry, the import and export of meat and other agricultural products, the consumption habits and consumption preferences of consumers, and the internal management and control level of the enterprise. The changing trends of various influencing factors can be shown through the analysis and description of relevant data[2]. Data is one of the important strategic resources owned and controlled by enterprises. For companies engaged in e-commerce marketing services, the importance of data analysis is even more self-evident. Through the analysis of industry big data, supply chain big data and consumer behavior and habits of enterprises, it can provide decision-making support for the managers of enterprises and improve the marketing effect. The specific process of data analysis is shown in Figure 1 below.

ISSN: 0010-8189

www.converter-magazine.info 

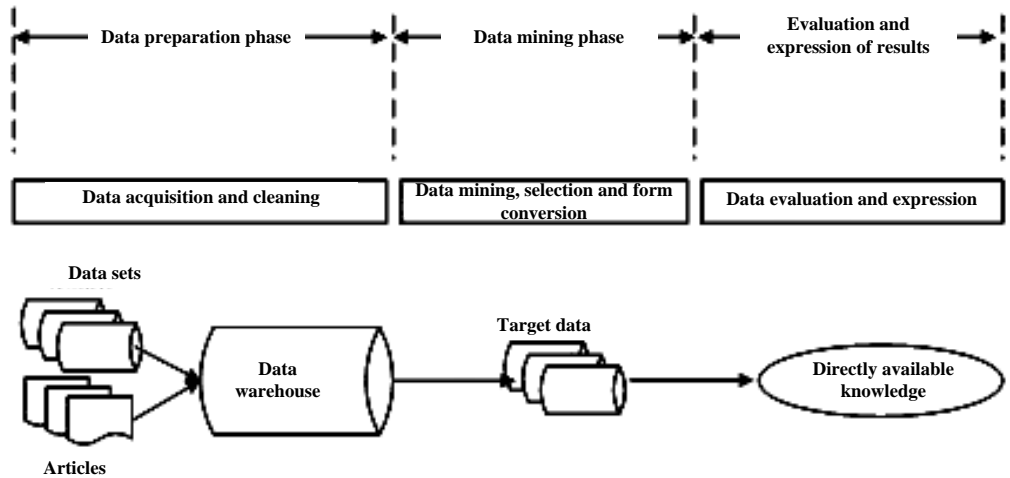

Figure 1. Stages and overall process of enterprise data analysis

Improve marketing capabilities based on data analysis:The decisions of the actors in the commodity market are generally in line with the laws of economic development and market changes. Centering on the supply-demand curve, the parties cooperate, compromise and play with each other. In the supply chain network system, it is an optimal economic state to achieve the balance of interests of all parties and maximize the interests of stakeholders. By means of industry data analysis, we can not only grasp the real external market conditions, but also the consumer preferences, and make marketing strategies according to the law of market changes and consumer demand preferences, so that we can obtain better marketing results. In the background of the Internet and e-commerce era, e-commerce enterprises can only obtain better market feedback by truly grasping market dynamics and taking personalized and precise marketing. In the context of the rapid development of network technology and communication technology, the marketing of enterprises is moving in the direction of informationization. The complex processes of enterprise operation, information exchange, and industry changes can be shown by the way of data changes. Through the collection and analysis of data, enterprise managers can gain insight into internal control issues, the general direction of the industry, and the latest features of the enterprise's product market, so that they can make scientific decisions and improve the marketing capabilities of e-commerce.

Innovative marketing models based on data analysis: In the context of the rapid development of the Internet and e-commerce economy, the marketing models of enterprises are no longer limited to a single model or a few models, and new marketing models are emerging, but whether these models can withstand the test of the market needs to be validated by simulation based on data analysis[2], in order to avoid more unanticipated losses from model innovation. First, the marketing model should be optimized at the level of improving the software database. With the help of big data framework and cloud computing tools, the most efficient and effective marketing model among existing models should be screened. Secondly, starting from analyzing big data of consumer consumption behavior, it should assist enterprise management in choosing the optimal marketing model. Thirdly, the marketing model should be innovated from the perspective of cost-profit relationship. The purpose of choosing a new marketing model is to make the enterprise e-commerce enterprise profitable, but the new marketing model has a great market risk. With the help of big data analysis tools and various numerical simulation software, simulation analysis from the cost-profit relationship can enable companies to establish contact with consumers in a virtual simulation environment, analyze the real needs of potential consumers, and determine the real relationship between cost and profit[3].

Enterprise industry big data and consumer big data contains a wealth of business information. Through data analysis, it can further reveal the development trend of the industry and the shopping habits of consumers, which is very important to promote the effect of personalized marketing for improving enterprise e-commerce or promoting the diversification of service models. However, in the process of data analysis, it is necessary to ensure that the data to be analyzed is continuous panel data, and the total amount of data should be large enough. With the help of big data 
framework and cloud computing tools, the function of data analysis can be better expressed[4].

\section{The development of personalized and precise marketing strategies of enterprises for e-commerce based on data analysis}

The personalized marketing strategy system of e-commerce based on data analysis consists of three aspects: product strategy, channel strategy and promotion strategy. The use of big data resources and data analysis means can help enterprises more accurately grasp the market dynamics, and then develop a more reasonable personalized marketing strategy.

Product marketing strategy Using the collected historical data on the marketing of a company's market products as the object of study, we redefine customer categories and the company's product system. Personalized marketing services are more directional because it refines customer groups based on data analysis and matches different product lines to each type of customer group for the purpose of personalized and precise marketing. In terms of the characteristics of corporate product customers, including large customers, fragmented customers and foreign customers, etc., personalized marketing programs matching the customer categories are developed for the characteristics of each type of customers. For large corporate customers, data analysis tools are used to grasp the order cycle, order variety and demand time of large customers in order to prepare raw materials in advance to shorten the delivery cycle. For general customers and customers with small demand, enterprises can use big data analysis software to integrate the resources of such customers and reasonably reserve inventory [5]. On the one hand, it can avoid the loss due to frequent multi-variety and small-lot production methods. On the other hand, it can also avoid excessive stock reserves that take up the enterprise's working capital. For overseas customers, enterprises should strictly implement the strategy of sales-based production and cash-on-delivery to avoid various risks from overseas markets[6].

Channel marketing strategy Channel marketing strategy is an important part of the personalized marketing strategy of enterprises. In terms of the marketing channels of enterprises in the context of rapid development of e-commerce mode, marketing channels mainly include online channels, offline channels and channels that integrate online and offline. Online transaction channel is the main channel of enterprise marketing activities, so enterprises should actively expand the scope covered by online channels and give priority to meeting the basic demands of large online customers. The predominance of online channels does not mean that offline channels are ignored. For the customers of the products of the majority of small and medium-sized enterprises, the offline channel is still a major shopping channel. In addition, offline stores can also be used as a place for online channel experience[7]. For the proportion of resources allocated to online and offline channels, the proportion of different customer categories can be analyzed with the help of data analysis tools to reasonably allocate marketing resources. The development mode of integrating online and offline channels is a channel marketing mode that is more helpful to the development of enterprise e-commerce. In the future,for enterprises online marketing is still the mainstream mode, but the boundary between online and offline marketing is more blurred and will gradually convert into an omnichannel marketing model [8].

Promotional marketing strategy From Keynes' Western economic theory, it is known that the decline in commodity prices has a significant pull on the demand curve. The Internet channel marketing model of enterprise goods can save a lot of marketing costs, transaction costs, logistics costs, etc. For enterprises, there is more room for concessions to consumers in the setting of commodity prices. Internet promotions have become a normal marketing method under the e-commerce model. Whether it is the double 11 shopping carnival led by Tmall Mall and Taobao, or the 618 shopping festival led by Jingdong, it occupies an important position in the total sales of e-commerce platforms throughout the year. From the big data survey and analysis of online consumers' consumption habits, it can be seen that most consumers are willing to choose the mode of concentrated consumption, because manufacturers' promotional marketing activities can bring more price concessions to consumers. In addition to online promotional activities, enterprises can also use invitation promotion to attract consumers with lower product prices. According to the inventory situation of the enterprise, product variety, the remaining shelf life of the product and other factors, the 
enterprise can issue an invitation to target consumers to give a lower market price of specific goods within a specified time. Regular sales promotions bring benefits to both buyers and sellers. Enterprises through promotional activities can reduce inventory occupancy, invigorate working capital, increase total sales, etc. Through concentrated consumption, enterprises can also significantly reduce the cost of commodity procurement and meet the basic shopping needs.

The above research results show that through the collection and analysis of market data, industry data and consumer data, the company's management can scientifically grasp the main features of the company's product marketing, channel marketing and product promotion marketing, and develop more reasonable personalized marketing programs for different potential customers.

\section{IV.Innovation of enterprise e-commerce marketing service model in the framework of data analysis}

Establish an e-commerce marketing service platform covering the entire enterprise product industry chain The Internet plays a fundamental role in the enterprise's e-commerce marketing system, and data resources such as supply chain data, industry data and consumer data are of great significance to the enterprise's e-commerce marketing. Therefore, the innovation of e-commerce marketing service model should be based around the Internet and big data. Enterprise industry supply chain big data shows that the current industry big data is not complete, and industry information does not play a full role in the enterprise product e-commerce marketing. Therefore, it is necessary to establish a kind of e-commerce marketing service platform covering the whole supply chain as soon as possible, and innovate the e-commerce service model. The supply chain e-commerce service platform integrates and innovates the original isolated service mode of each link of the supply chain, and integrates order management, tracking management, storage management and distribution management in one. In this way, it can reduce the circulation cost of the original enterprise products and shorten the delivery cycle of goods at the same time. After the consumer places an order and it is confirmed, the order information will be transmitted to each node enterprise in the supply chain after data analysis. The system will monitor the entire order flow process, and each supply chain subject, including the user, can grasp the order dynamics in real time. The logistics system based on data analysis can automatically allocate the logistics mode and the logistics and transportation management of goods according to the delivery cycle of orders and the size of orders, and the centralized and unified scheduling is more conducive to the allocation and integration of logistics resources and the improvement of service quality for users.

Build an e-commerce marketing service evaluation system: In order to give consideration to fairness and justice in the process of enterprise product marketing, the innovation of e-commerce marketing service mode also lies in the ability to score enterprises according to users' real feelings. The scoring and inspection results of enterprises can be queried through the supply chain system, forming a mechanism of survival of the fittest, and gradually becoming an important basis for users to select customers. The purpose of the enterprise's personalized marketing service mode is to provide the end users with better industry and service, and the merits and disadvantages of the e-commerce service mode should ultimately be evaluated by consumers. Consumers' evaluation of supply chain efficiency and their evaluation and complaint of enterprise service level will be the direct impetus for enterprises to carry out reform. With the support of big data analysis, the e-commerce service evaluation system can more objectively and truly reveal the shortcomings of existing service modes, and help enterprises to make continuous improvement.

Improve product traceability and after-sales service of enterprise products: Doing a good job of after-sales service is an important part of improving the e-commerce service model of the company. Many customer complaints are caused by the imperfect and unsound after-sales service system. For special commodities like enterprise products, once problems occur, they will cause serious losses to the livestock industry, so the traceability of commodities plays a crucial role. Based on the framework of big data, every batch of goods should be included in the e-commerce information service platform by means of data analysis. Once there is a problem with the product, the enterprise can quickly find out the batch of goods and formulate emergency measures in a targeted manner. The traceability management of enterprise e-commerce products covers many aspects such as raw material procurement, product

ISSN: 0010-8189 
processing and manufacturing, warehousing and logistics and terminal sales, etc. The total amount of data is huge, so it is only possible to resort to the means of data analysis. Consumers can check the flow trajectory of goods in the whole process of the supply chain through the e-commerce platform, and even visualize product archiving for enterprise products, which helps improve the quality of goods.

\section{Conclusion}

Enterprise commodity e-commerce marketing has become an important way of enterprise marketing. However, the e-commerce model will face such problems as too much marketing data, missing or incomplete data, and inability to accurately grasp consumers' demand preferences. For the problem of e-commerce marketing in the background of Internet big data era, this paper builds a personalized marketing system based on data analysis, and innovates the service mode of e-commerce from the perspectives of building an e-commerce information service platform, building an e-commerce service evaluation system, and improving after-sales service, so as to provide better after-sales service for users.

\section{Acknowledge}

This research was supported byA research of tactics and policy of developing "Incubation \& Venture capital investment" Entrepreneurship Model's Specialization Maker Space in Ningbo city ,China ( 2017A10003), Soft Science Project in Ningbo in 2017.

\section{Reference}

[1] $\mathrm{Hu}$ Yi. Research on personalized information recommendation service model of e-commerce based on big data [D]. Jilin University

[2] Chen Jian. Analysis on the current situation of e-commerce marketing methods of small and medium-sized enterprises in China [J]. China business theory, 2011

[3] LV Zhao, Gu Junzhong. E-commerce platform model supporting collaboration and intelligent decision-making [J]. Computer engineering and design, 2002, 023 (002): 21-25

[4] Huang Jialiang, Gu bin. E-commerce industry supervision system based on big data [J]. China Science and Technology Forum, 2016 (5): 46-51

[5] Liu Yijin, Huang Xiaohong. Research on budget management of e-commerce enterprises in the era of big data [J]. Journal of Changchun University, 2016, 026 (002): 25-29

[6] Rong feiqiong, Guo Mengfei. Research on supplier credit evaluation of cross-border e-commerce platform based on big data [J]. Statistics and information forum, 2018

[7] Du Danqing. E-commerce diversification and retail market reform in the era of big data [J]. Economist, 2014 (11): 102-104

[8] Huang Hailong. Research on Internet Finance Based on e-commerce platform [J]. Shanghai finance, 2013, 000 (008): 18-23 